\title{
Some Aspects of Rectangular Microstrip Antenna for Mobile Application
}

\author{
Prabir Banerjee $^{1}$ and Tulshi Bezboruah ${ }^{2}$ \\ ${ }^{I}$ Department of Physics, Jagiroad College, Jagiroad-782410, Morigaon, Assam, INDIA, \\ Tel: $+91-3678-242308(O)$ \\ ${ }^{2}$ Department of Electronics \& Communication Technology, Gauhati University, Guwahati- \\ 781014, Assam, INDIA, Tel:+91-361-2671262(O), Fax: +91-361-2700311(O), \\ prabirjrd1962@gmail.com ${ }^{1}, z b t \_g u @ y a h o o . c o . i n^{2}$
}

\begin{abstract}
We investigate the effects of antenna parameters on the radiation characteristics of a compact size Rectangular Microstrip Antenna suitable for mobile application. In the calculations of antenna parameters, resonant frequency $f_{r}$ is set at $3 \mathrm{GHz}$. In our investigations three low cost substrate materials, namely: (a) irradiated poly-guide with relative dielectric constant and loss tangent as $\epsilon_{r}=2.32$, $\tan \delta=0.0005$, (b) Glass-Epoxy with $\epsilon_{r}=3.7, \tan \delta=0.02$ and (c) R03006 with $\epsilon_{r}=6.15, \tan \delta=0.0013$ are used to simulate the antenna parameters obtained from various models. The simulation results predict that the $B W$ increases with the increase in substrate thickness and decrease in dielectric constant, while directivity strongly depends on width of the patch. Here we will present in details the antenna model, its simulation and the results of our analysis.
\end{abstract}

Keywords: Dielectric constant, Loss tangent, Rectangular Microstrip antenna, Resonant frequency

\section{Introduction}

In communication systems, a major challenge is the design of a compact size antenna which can provide wideband characteristic over the entire range of operating bandwidth (BW). The printed circuit design of antennas also popularly known as Microstrip Antenna(MSA) are very attractive design for short and long range communications, such as, aerospace and missile technology due to its low profile, light weight, compact size, conformable to mount structure, easy fabrication and can also be integrated with solid-state devices. It can also be integrated with hand-held devices such as cell and cordless phones. Descamp and Gutton et al. [1-2] proposed the model of MSA for the first time. The demands of MSA have been increasing rapidly, especially within the past two decades, because of its unique features. Although the MSA is generally known for its shortcomings of narrow $\mathbf{B W}$, recent technological advances have paved the way for broadband applications. With the aid of different feeding techniques, meandering, electronic tuning circuits with active components, it has become possible to increase BW from a few percent to tens of percent. First attempt to design of MSA started with the work of Muson [3] and followed by Howell, Derneryd, Carver, Kerr and Huan [4-8]. With the increasing demand from the industries, there was extensive research works carried out for the development of wide band MSA [9-10].

The objective of our proposed work is to model and simulate key antenna parameters with MATLAB simulation software, necessary to design a compact size rectangular microstrip antenna (RMSA) suitable for handheld devices. We investigate the same by considering the 
parameters namely: (i) effective dielectric constant, (ii) length extension, (iii) $\mathbf{B W}$, (iv) directivity, (v) radiation efficiency and (vi) effects of finite ground plane (GP) on resonant frequency.

\subsection{Related Works}

In the year 1978, Anders G. Derneryd [5] theoretically investigated the rectangular microstrip antenna as a two slot model and calculated input impedance, mutual conductance and directivity of the antenna.

In the year 1991, David R. Jackson and Nicolaos G. Alexopoulos [15] derived simple formulas for resonant input resistance, BW, and radiation efficiency of a rectangular microstrip patch on a thin substrate. Although their formulas are approximate, the BW and efficiency formulas are sufficiently accurate and may be used for final design purposes.

In the year 2010, Rajesh Kumar Vishwakarma and Sanjay Tiwari [9] proposed a stacked rectangular microstrip antenna that can be used for mobile applications.

In 2011, K. J. Vinoy [16], proposed a multi-functional microstrip patch with two U shaped slots antennas for the primary application in wireless systems which virtually cover entire commercial wireless bands.

In the year 2012, R. D. Kanphade, D. G. Wakade and N.T. Markad [17] proposed a rectangular Microstrip patch antenna for satellite communication.

\section{Design Considerations}

The MSA design follows the microstrip transmission line theory. According to this theory the MSA can be represented by four apertures [11]. Out of these two of them are radiating slots while other two are non-radiating. We consider the radiating slots model, since they accounts for most of the radiation from the antenna. This model simplifies MSA as a lossless parallel-plated transmission line separated by length $\mathbf{L}$ and each of the slots having width $\mathbf{W}$ and height $\mathbf{h}$ [11]. The MSA is an interface of two media air and Substrate. Two waves have different propagation constant in air and substrate so effective dielectric constant has to be considered to account for fringing length extension as it influences the resonant frequency [11]. We take the simple rectangular configuration for the analysis of the patch antenna with length $\mathrm{L}$ in the range of $\lambda_{0} / 3<L<\lambda_{0} / 2$, where $\lambda_{0}$ is the wavelength in air [11]. The thickness of the patch metallization is considered to be negligible compared to the wave length in air medium [11]. The height of the dielectric substrate is taken in the range $\mathbf{0 . 0 0 3} \boldsymbol{\lambda}_{\mathbf{0}} \leq \boldsymbol{h} \leq \mathbf{0 . 0 5} \boldsymbol{\lambda}_{\mathbf{0}}$, since surface wave power increases with thick dielectric substrate [11]. However, for $\boldsymbol{h} \leq \mathbf{0 . 3} \lambda_{0} / \mathbf{2} \pi \sqrt{\epsilon_{r}}$ the antenna loss associated with surface wave can be neglected [12]. The relative permittivity of the substrate $\boldsymbol{\epsilon}_{r}$ is taken to be $\mathbf{2 . 3 2} \leq \boldsymbol{\epsilon}_{r} \leq \mathbf{6 . 1 5}$. The ratio between copolarization to cross-polarization component attains maximum value, when aspect ratio W/L is 1.5. Therefore, to suppress the cross polarization component, aspect ratio should satisfy the condition $\mathbf{W}=\mathbf{1 . 5} \mathbf{L}$. The MSA is broadside radiator. The efficiency of MSA is an inverse function of substrate dielectric constant and thickness $\mathbf{h}$ so a small value of which lead to higher efficiency. Miniaturization of MSA could be achieved by using substrate with higher value of relative permittivity at the cost of $\mathbf{B W}$ and efficiency. So, one need to compromise among antenna size, efficiency and $\mathbf{B W}$, in order to achieve the desired goal.

\section{Method of Analysis}

The Patch antennas can be modeled as a simple two slots transmission line or dielectric loaded cavity [11]. Both the models are useful for analysis of planar structures and are most 
popular among the antenna designers. The computed pattern, input impedance and resonant frequencies of cavity model analysis of RMSA are found to be consistent with the measured results as reported in [11]. We concentrate our analysis on both the models.

\subsection{Transmission Line Model}

The equivalent representation of RMSA is shown in Figure 1. Due to finite dimensions of MSA, the fields at the edge of patch along the length and width undergo fringing. As result electrical length becomes slightly more than the physical length [11]. The fringing extension also applies for width; however effect of length extension can substantially influence resonant frequency and wave propagation velocity [11]. An effective dielectric constant $\left(\boldsymbol{\epsilon}_{\text {ef }}\right)$ has values in the range of $\mathbf{1} \leq \epsilon_{\text {ef }} \leq \epsilon_{r}$ [11].The effective dielectric constant depends upon substrate thickness and frequency. At lower frequency relative permittivity is almost constant. The low frequency approximation of $\boldsymbol{\epsilon}_{\text {ef }}$ for thin substrate can be expressed as [11]:

$$
\epsilon_{e f}=\frac{\left(\epsilon_{r}+1\right)}{2}+\frac{\left(\epsilon_{r}-1\right)}{2}\left[1+12 \frac{h}{W}\right]^{\frac{1}{2}}
$$

where $\epsilon_{r}$ is the relative permittivity of substrate material, $\mathbf{h}$ is thickness of the substrate and W is microstrip line width.

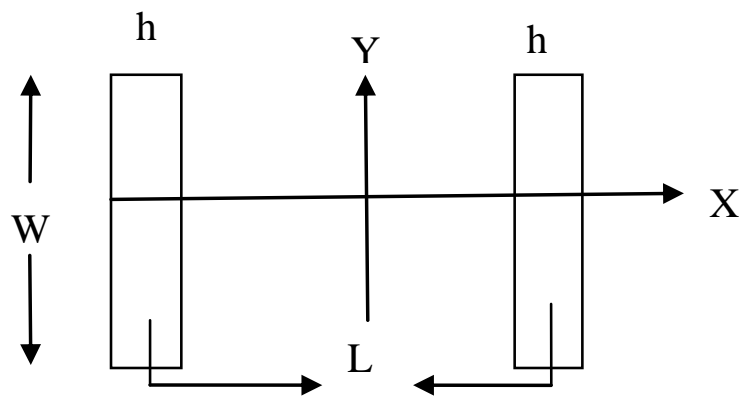

Figure 1. Two Slot Transmission Line Model of RMSA

The improved expression for length extension given in [13] as:

$$
\Delta L=h \xi_{1} \xi_{3} \xi_{5} / \xi_{4}
$$

Where $\xi_{1}=0.434907 \frac{\epsilon_{\text {reff }}^{\text {usp }}+0.26}{E_{\text {reff }}^{0.81}-0.189} \frac{(W / h)^{0.8544}+0.236}{(W / h)^{0.8544}+0.87}$

$\xi_{2}=1+\frac{(W / h)^{0.371}}{2.358 \epsilon_{r}+1}, \quad \xi_{3}=1+0.5274 \arctan \left[0.084(W / h)^{1.9413 / \xi_{2}}\right] / \epsilon_{\text {reff }}^{0.9236}$

$\xi_{4}=1+0.0377 \arctan \left[0.067(W / h)^{1457}\right] \times\left\{6-5 \exp \left[0.036\left(1-\epsilon_{r}\right)\right]\right\}$

$\xi_{5}=1-0.218 \exp (-7.5 \mathrm{~W} / \mathrm{h})$

The effective length and width is given [11]

$$
L_{e f}=L+2 \Delta L
$$




$$
W=0.15 / f \sqrt{\left(\epsilon_{r}+1\right) / 2}
$$

where $\mathrm{f}=$ frequency in $\mathrm{GHz}$

\subsection{Effect of Finite Ground Plane}

The finite GP gives rise to diffraction of radiation at the periphery of the bottom conductor, resulting in change in radiation pattern, radiation conductance and resonance frequency. If patch size metallization of an antenna is equal to the dimension of the GP, the resonant frequency is higher compared to that of an infinite sized GP antenna. Denoting GP extension by $\mathbf{d}$, the fractional change in resonant frequency is given by [12]:

$$
\frac{\Delta f}{f_{r}}=\frac{1}{\pi} \frac{E_{r}+1}{\sqrt{E_{r}}} \frac{h}{\lambda_{0}} \ln \left(\frac{W}{h}\right), k_{0} d \rightarrow 0 \text { for } \frac{W}{h} \gg 1
$$

For other value of $\mathbf{d}$ not satisfying the limit the expression can be written as:

$\Delta \boldsymbol{f} / \boldsymbol{f}_{r}=-240 \mathrm{~g}_{d} /\left(\sqrt{\epsilon_{e f}} \boldsymbol{W}\right)$

With $g_{d}=\frac{k_{0} W}{8 \eta_{0}}\left[J_{0}^{2}\left(k_{0} d\right)-Y_{0}^{2}\left(k_{0} d\right)\right]$

where $\boldsymbol{J}_{0}$ and $\boldsymbol{Y}_{\mathbf{0}}$ are zero order Bessel function of first and second kind and $\eta_{0}$ is characteristics impedance of free space.

The ground plane dimensions are given as:

$$
\begin{aligned}
& L_{g}=5.8 h+\mathrm{L} \\
& W_{g}=5.8 h+\mathrm{W}
\end{aligned}
$$

\subsection{Cavity Model}

Although the transmission line model is simple, it can be advantageous for design of patches of planar shape, but the field between the patch and the ground plane are not taken into account. By assuming MSA to be a lossless cavity, it would not radiate power; to sustain radiation loss mechanism has to be included. The cavity model is constructed, based upon the assumption that, the patch and ground plane acts as electric conducting walls and surrounded by magnetic walls along the perimeter of the model space. Figure 2(a) shows the cavity model of the RSMA while Figure 2(b) shows the top view of field and magnetic current distribution. According to thin substrate approximation, the field variation along the height is assumed to be constant, in the direction perpendicular to the patch [11].

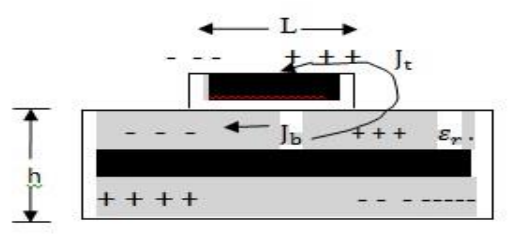

Figure 2 (a). Cavity Model of RMSA 


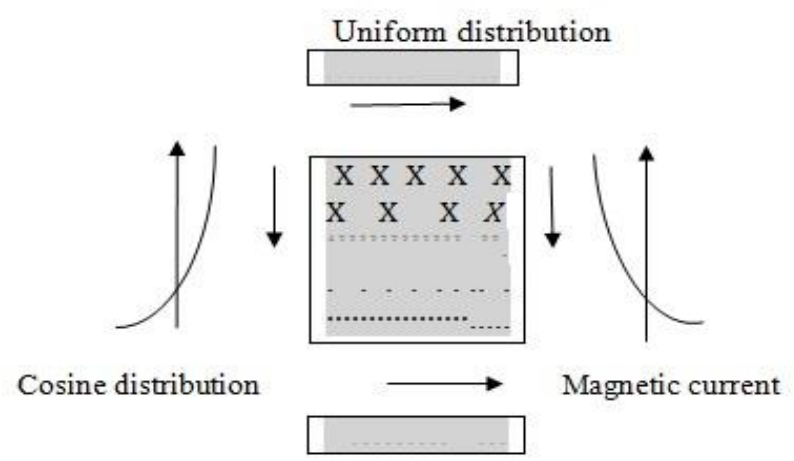

Figure 2 (b). Top View of Field and Magnetic Current Distributions

\subsection{Radiation Efficiency}

The propagation of surface wave in addition to dielectric and conductor loss for thin substrate approximation has profound effect on efficiency of patch antenna. The radiation efficiency, without accounting into dielectric and conduction losses can be expressed as [12]:

$$
\mathrm{e}_{\mathrm{r}}^{0}=\mathbf{P}_{\mathbf{r}} /\left(\mathbf{P}_{\mathbf{r}}+\mathbf{P}_{s w}\right)
$$

where

$$
\begin{gathered}
\mathbf{P}_{\mathbf{r}}=40 \mathrm{k}_{0}{ }^{2}\left(\mathrm{k}_{0} \mathbf{h}\right)^{2} \mathbf{c}_{1} \\
\mathbf{P}_{\mathrm{sW}}=30 \pi \mathrm{k}_{0}{ }^{2} \epsilon_{r} X /\left(\epsilon_{r}\left[\frac{1}{\sqrt{X}}+\frac{\sqrt{X}}{Y}\right]+\left(k_{0} h\right)\left[1+\frac{\epsilon_{T}^{2} X}{Y}\right]\right. \\
\boldsymbol{c}_{1}=1-\frac{1}{n_{1}^{2}}+\frac{2 / 5}{n_{1}^{4}}, n_{1}=\sqrt{\epsilon_{r} \mu_{r}} \\
\left.\left.\mathrm{X}=x_{0}{ }^{2}-1, \mathrm{Y}=\epsilon_{r}-x_{0}{ }^{2} \text {, and } x_{0}=1+0.5 *\left(\epsilon_{r}-1\right) * \mathbf{k} * \mathbf{h} / \epsilon_{r}\right)^{2}\right)
\end{gathered}
$$

The radiation efficiency for a horizontal electric dipole model can be expressed in terms of $\mathbf{P}_{\mathrm{sp}}^{\mathrm{HED}}$ power radiated into space by a unit strength horizontal electric dipole on the lossless substrate and $\mathbf{P}_{\mathbf{s W}}^{\mathrm{HED}}$ the power radiated into surface wave by the dipole as [14]:

$e_{r}^{H E D}=\frac{\mathrm{P}_{\mathrm{Sp}}^{\mathrm{HED}}}{\mathrm{P}_{\mathrm{sp}}^{\mathrm{HED}}+\mathrm{P}_{\mathrm{sW}}^{\mathrm{HED}}}$

Where $\mathbf{P}_{\mathrm{sp}}^{\mathrm{HED}}=\frac{1}{\boldsymbol{Z}_{0}^{2}}\left(\boldsymbol{k}_{0} h\right)^{2}\left(\mathbf{8 0} \pi^{2} \mu_{r}^{2} c_{1}^{\prime}\right)$

$c_{1}^{\prime}=\frac{1}{n_{1}^{2}}+\frac{2 / 5}{n_{1}^{4}}$, and $n_{1}=\sqrt{\epsilon_{r} \mu_{r}}$

$\mathrm{P}_{\mathrm{sW}}^{\mathrm{HED}}=\frac{1}{z_{0}^{2}}\left(k_{0} h\right)^{3}\left(60 \pi^{3} \mu_{r}^{3}\left(1-\frac{1}{n_{1}^{2}}\right)^{3}\right)$ 
The improved expression for $\mathbf{P}_{\mathbf{s W}}^{\mathrm{HED}}$ with higher order terms in the approximation for nonmagnetic substrate $\left(\boldsymbol{\mu}_{\boldsymbol{r}}=\mathbf{1}\right)$ can be expressed as [14]:

$\mathbf{P}_{\mathrm{sW}}^{\mathrm{HED}}=\frac{\eta_{0} k_{0}^{2}}{8} \frac{E_{\mathrm{r}}\left(x_{0}^{2}-1\right)^{3 / 2}}{E_{\mathrm{r}}\left[x_{1}+1\right]+\left(k_{0} h\right)\left[E_{r}^{2} x_{0}+1\right] \sqrt{x_{0}^{2}-1}}$

where $\boldsymbol{x}_{1}=\frac{x_{0}^{2}-1}{E_{r}-x_{0}^{2}}, x_{0}=1+\frac{-E_{r}^{2}+\alpha_{0} \alpha_{1}+E_{r} \sqrt{E_{r}^{2}-2 \alpha_{0} \alpha_{1}+\alpha_{0}^{2}}}{E_{r}^{2}-\alpha_{1}^{2}}, \alpha_{0}=s \tan \left(k_{0} h s\right)$,

$s=\sqrt{\epsilon_{r}-1}$,

and $\quad \alpha_{1}=-\frac{1}{s}\left[\tan \left(k_{0} h s\right)+\frac{k_{0} h s}{\cos ^{2}\left(k_{0} h s\right)}\right]$

The Computer Aided Design (CAD) formula of radiation efficiency for horizontal electric dipole model of MSA can be expressed as [14]:

$e_{r}^{C A D}=e_{r}^{H E D} /\left(1+e_{r}^{H E D}\left[\delta_{e f}+\left(\frac{R_{s}}{\pi \eta_{0} \mu_{r}}\right)\left(\frac{1}{h_{/ / \lambda_{0}}}\right)\right]\left[Q_{s p}^{C A D}\right]\right)$

The resistance due to skin effect on surface of the patch and ground plane metallization at frequency $\boldsymbol{\omega}=\mathbf{2} \boldsymbol{\pi} \boldsymbol{f}$, is [14]:

$R_{s}=\sqrt{\omega \mu_{0} / 2 \sigma_{c}}$

where $\sigma_{c}$ is the conductivity of the patch metallization and $\delta_{e f}$ is the effective loss tangent defined as [14]:

$\delta_{e f}=\left[\tan \delta+\left(\frac{R_{S}}{\pi \eta_{0} \mu_{r}}\right)\left(\frac{1}{\frac{h}{\lambda_{0}}}\right)+\left(\frac{16}{3}\right)\left(\frac{p c_{1}}{\epsilon_{r}}\right)\left(\frac{h}{\lambda_{0}}\right)\left(\frac{W_{e}}{L_{e}}\right)\left(\frac{1}{e_{r}^{S W}}\right)\right]$

The space-wave $\mathbf{Q}$-factor formula in terms of CAD is given by [14]:

$Q_{s p}=\frac{3}{16}\left(\frac{E_{r}}{p c_{1}}\right)\left(\frac{L_{e f}}{W_{e f}}\right)\left(\frac{1}{h / \lambda_{0}}\right)$

with

$p=1+\frac{a_{2}}{10}\left(k_{0} W_{e}\right)^{2}+\left(a_{2}^{2}+2 a_{4}\right)\left(\frac{3}{560}\right)\left(k_{0} W_{e}\right)^{4}+c_{2} \frac{1}{5}\left(k_{0} L_{e}\right)^{2}+a_{2} c_{2}\left(\frac{1}{70}\right)\left(k_{0} W_{e}\right)^{2}\left(k_{0} L_{e}\right)^{2}$

$c_{1}^{\prime}=1-\frac{1}{n_{1}^{2}}+\frac{2 / 5}{n_{1}{ }^{4}}$, and $n_{1}=\sqrt{\epsilon_{r} \mu_{r}}$

\subsection{Bandwidth}

The $\mathbf{B W}$ is a strong function of substrate height $\mathbf{h}$ and maintains direct proportionality provided, dielectric, conductor, and surface-wave losses are negligible. The higher-order modes are exited for thicker substrate and Q of the cavity is also decreases, at the same time feed radiation also increases significantly [14].Matching becomes difficult for thicker substrate beyond the range of inequality $\boldsymbol{h} \geq \mathbf{0 . 0 5} \boldsymbol{\lambda}_{0}$. The BW is defined with respect to a minimum value of Voltage Standing Wave Ratio (VSWR). 
The BW equation in terms of Computer Aided Design formulation with Voltage Standing Wave Ratio (VSWR) below less than equal to two can be expressed as [14]:

$\mathbf{B W}=\frac{1}{\sqrt{2}}\left[\tan \delta+\left(\frac{R_{s}}{\pi \eta \mu_{r}}\right)\left(\frac{1}{\frac{h}{\lambda_{0}}}\right)+\left(\frac{16}{3}\right)\left(\frac{p c_{1}}{\epsilon_{r}}\right)\left(\frac{h}{\lambda_{0}}\right)\left(\frac{W_{e}}{L_{e}}\right)\left(\frac{1}{e_{r}^{S W}}\right)\right]$

If dielectric and conductor losses are neglected the $\mathbf{B W}$ formula simplifies to:

$$
\mathrm{BW}^{0}=\frac{1}{\sqrt{2}}\left[\left(\frac{16}{3}\right)\left(\frac{p c_{1}}{\epsilon_{\mathrm{r}}}\right)\left(\frac{h}{\lambda_{0}}\right)\left(\frac{W_{e}}{L_{e}}\right)\left(\frac{1}{e_{r}^{S W}}\right)\right]
$$

\subsection{Directivity of RMSA}

The CAD formula for directivity is given as [15]:

$D_{0}=\left(\frac{\eta_{0}}{40 \pi}\right)\left(\frac{1}{p c_{1}}\right)\left[\frac{\frac{\tan ^{2}\left(k_{0} h n_{1}\right)}{\left(k_{0} h n_{1}\right)^{2}}}{1+\left(\frac{\mu_{r}}{\epsilon_{\mathrm{r}}}\right) \tan ^{2}\left(k_{0} h n_{1}\right)}\right]$

If the substrate is electrically thin then the directivity formula reduced to:

$$
D_{0}=\left(\frac{\eta_{0}}{40 \pi}\right)\left(\frac{1}{p_{c 1}}\right)
$$

Single slot directivity formula relating to width of free space wavelength is given as [11]:

$D_{0}=\left(\frac{2 \pi W}{\lambda_{0}}\right)^{2} \frac{1}{I_{1}}$

where

$I_{1}=\int_{0}^{\pi}\left[\frac{\sin \left(k_{0} W / 2 \cos \theta\right)}{\cos \theta}\right]^{2} \sin ^{3} \theta d \theta$

$=\left[-2+\cos X+X S_{i}(X)+\frac{\sin X}{X}\right], X=k_{0} W$, and $S_{i}(X)=$ Sine integral.

\section{Design of RMSA and Simulation}

Based on various models we calculate resonant length, width of patch, substrate height, effective dielectric constant, radiation efficiency, $\mathbf{B W}$, directivity and dimension of $\mathbf{G P}$ as shown in the Table-I, II, III and IV. The GP dimensions for three antennas are as given below:

(i) Antenna \#1 for $\epsilon_{r}=\mathbf{2 . 3 2}$, Length $64 \mathrm{~mm}$, width $89.6 \mathrm{~mm}$, substrate thickness 3.1 $\mathrm{mm}$

(ii) Antenna \#2 for $\epsilon_{r}=\mathbf{3 . 7}$, Length $\mathbf{6 2 . 2} \mathrm{mm}$, width $\mathbf{8 7 . 2} \mathrm{mm}$, substrate thickness $2.5 \mathrm{~mm}$

(iii) Antenna \#3 for $\epsilon_{\boldsymbol{r}}=\mathbf{6 . 1 5}$, Length $\mathbf{5 9 . 5} \mathrm{mm}$, width $84.5 \mathrm{~mm}$, substrate thickness 1.9 $\mathrm{mm}$ 
Simulation of eqns. (2), (5), (6), (9) (11) (12) (12a), (13) and (14) is carried out on MATLAB Platform version 7.1 to study the various characteristics of the antenna models.

\section{Results and Discussions}

We have calculated effective dielectric constants of three different substrate materials as listed in Table 1. We simulate fringing length extension eqn. (2) as function of substrate thickness and width to $\mathrm{h}$ ratio $(\mathrm{W} / \mathrm{h})$ and the responses are shown in Figure 3-4. It is observed that fringing length increases sharply with increase in substrate thickness while for other plot as $\mathrm{W} / \mathrm{h}$ ratio increase fringing length extension remain constant since increase in $\mathrm{W} / \mathrm{h}$ ratio means decrease in substrate thickness and hence fringing length extension remain constant.

\section{Table 1. Calculation of effective dielectric constant and Length extension $(\Delta L)$, design length $\left(L^{\prime}\right)$ and width $\left(W^{\prime}\right)$ of patch at $\mathrm{f}=3 \mathrm{GHz} \in_{\text {ef }}$ for Substrate height $\mathrm{S} \leq .0477 \lambda_{0} / \sqrt{\epsilon_{r}}, \mathrm{t}=0.3 \mathrm{~mm}$}

\begin{tabular}{|c|c|c|c|c|c|c|c|c|}
\hline $\begin{array}{l}\text { SL. } \\
\text { No }\end{array}$ & $E_{r}$ & h $\mathrm{mm}$ & $E_{e f}$ eqn.(1) & $\Delta$ Lmmeqn, (2) & L mm & W mm & $L^{s} \mathrm{~mm}$ & $W^{s} \mathbf{m m}$ \\
\hline 1 & 2.32 & 3.1 & 2.1986 & 2 & 50 & 75 & 46 & 71 \\
\hline 2 & 3.7 & 2.5 & 3.4921 & 1.4 & 50 & 75 & 47.2 & 72.2 \\
\hline 3 & 6.15 & 1.9 & 5.8265 & 0.94 & 50 & 75 & 48.1 & 73.1 \\
\hline
\end{tabular}

The effect of finite GP on antenna resonant frequency eqn. (5-6) as a function of normalized substrate thickness and extended length beyond patch metallization in all sides is simulated and the responses are shown in Figure 5 (a)-5(b). It is observed that as the size of the GP is increased, the resonant frequency decreases and approaches to that of the infinite size GP when the increase in of GP size is about $\mathbf{d}=\mathbf{0 . 0 3 5} \boldsymbol{\lambda}_{0}$ on all sides. As the size of GP is increased, the fractional change in resonant frequency decreases to that of infinite GP value. From the minimum value of fractional change in resonant frequency we can calculate the required dimension of GP. The condition for infinite GP is satisfied Figure 5 (a) if substrate thickness is $\mathbf{h} \geq \mathbf{0 . 0 7 5} \boldsymbol{\lambda}_{0}$. The GP dimension extend in all side of patch metallization satisfying the inequality $\mathbf{d} \geq \mathbf{0 . 0 3 5} \boldsymbol{\lambda}_{0}$, the fractional change in resonant frequency becomes zero value as shown in Figure 5(b). Radiation Efficiency for a rectangular patch antenna without accounting losses due to substrate material and losses for skin effect and ohmic loss on patch metallization represented by eqn. (9) is simulated by MATLAB based simulator and the response is depicted in Figure 6 (a). At operating frequency accounting dielectric and conductor losses eqn. (11) is simulated and the response is shown in Figure 6 (b). The dielectric and conductor losses, increases substantially for substrate thickness is $\boldsymbol{h}<\boldsymbol{\lambda}_{\mathbf{0}} / \mathbf{5 0}$ while for $\boldsymbol{h}>\boldsymbol{\lambda}_{\mathbf{0}} / \mathbf{5 0}$, the losses due to surface-wave propagation becomes significant. Excluding the effect of dielectric and conductor losses on antenna, radiation efficiency would be almost $\mathbf{1 0 0 \%}$, and with increase in the substrate thickness, efficiency fall sharply. From the simulation of BW, accounting losses eqn. (12) and without losses eqn. (12a) vs normalized substrate thickness $\left(\mathbf{h} / \boldsymbol{\lambda}_{0}\right)$, moderate to high value results the responses as shown in Figure 7(a)-7(b). It is observed that the BW increases substantially. From Figure 7 (a), it is apparent that BW that is a strong function of height of the dielectric substrate $\boldsymbol{h}$ and aspect ratio (W/L). Calculated $\mathbf{B W}$ is given in the Table 2. It is observed that $\mathbf{B W}$ improvement could be achieved among others by proper selection of supporting substrate material with low value dielectric constant and higher values of aspect ratio, higher value of loss tangent, or by increasing the substrate height. The simulation of directivity eqn. (13) with aspect ratio 
$W_{e} / L_{e}=1.5$ and three different substrate material of relative permittivity $\boldsymbol{\epsilon}_{r}=\mathbf{2 . 3 2}, \boldsymbol{\epsilon}_{r}=\mathbf{3 . 7}$ and $\epsilon_{r}=6.15$ is carried out and the response is shown in Figure 8(a).

Table 2. Comparison of Radiation Efficiency $\left(e_{r}\right)$ for Substrate Height $\mathbf{h}(1.9 \mathrm{~mm}$ $-3.1 \mathrm{~mm})$ for Different Dielectric Constant $\left(E_{r}\right)$ and Loss Tangent $(\tan \delta)$

\begin{tabular}{|l|l|l|l|l|l|l|}
\hline $\begin{array}{l}\text { SL. } \\
\text { No. }\end{array}$ & $\in_{r}$ & $\tan \delta$ & $\mathbf{h ~ m m}$ & $\mathbf{e}_{\mathbf{r}}^{0} \%$ eqn.(9) & $e_{r}^{H E D} \%$ eqn.(10) & $e_{r}^{C A D} \%$ eqn.(11) \\
\hline 1 & 2.32 & 0.0005 & 3.1 & 95.59 & 98.22 & $\mathbf{8 8 . 3 8}$ \\
\hline 2 & 3.7 & 0.02 & 2.5 & 86.82 & 96.62 & 84.21 \\
\hline 3 & 6.15 & $\mathbf{0 . 0 0 1 3}$ & 1.9 & $\mathbf{7 1 . 6 0}$ & $\mathbf{9 5 . 6 5}$ & $\mathbf{8 3 . 5 1}$ \\
\hline
\end{tabular}

We also simulate variation of directivity eqn. (14) considering normalized width $W / \boldsymbol{\lambda}_{\mathbf{0}}$ and the response is shown in Figure 8(b). From the response it is observed that directivity is a strong function of patch width and dielectric constant.

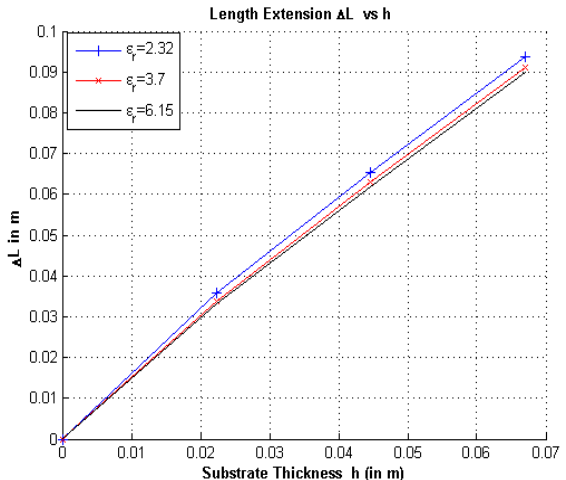

Figure 3. Fringing Length Extension eqn. (2) vs. Substrate Thickness $h$ (in m) for Three Different Dielectric Materials

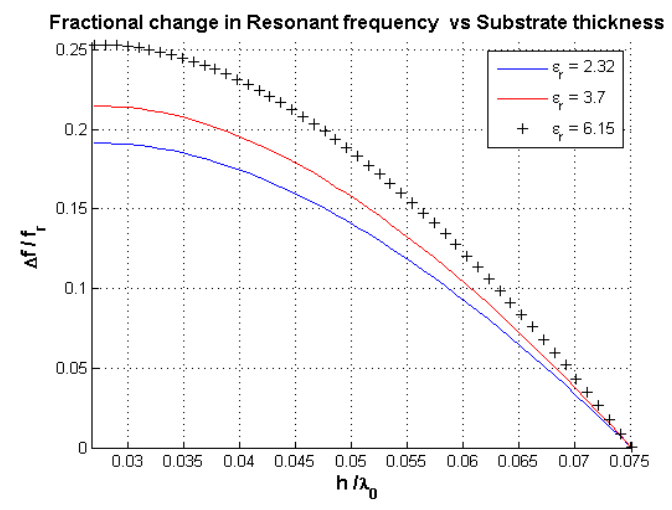

Figure 5(a). Fractional Change in Resonant Frequency eqn.(5) as a Function of Normalized Substrate Thickness (h) for $E_{r}=2.32,3.7 \& 6.15$

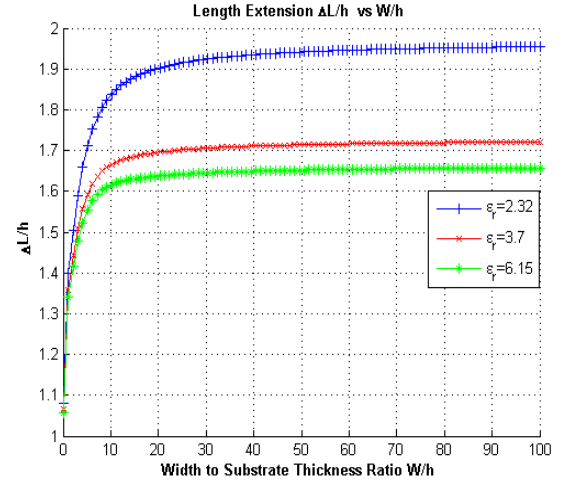

Figure 4. Fringing Length Extension eqn. (2) vs. Width to Substrate Thickness Ratio (w/h) for Three Different Dielectric Materials

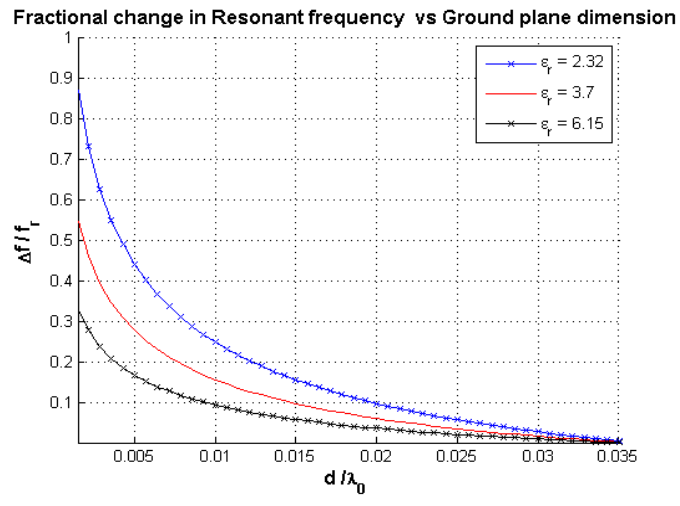

Figure 5(b). Fractional Change in Resonant Frequency eqn.(6) as a Function of Normalized Extended Length from All Side of Patch (d) 


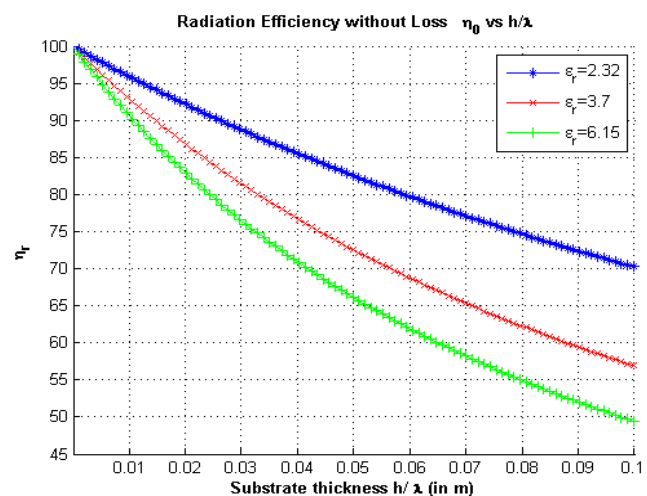

Figure 6(a). Radiation Efficiency eqn.

(9) as a Function of Substrate

Thickness (h) for $\epsilon_{r}=2.32,3.7 \& 6.15$ without Dielectric Losses

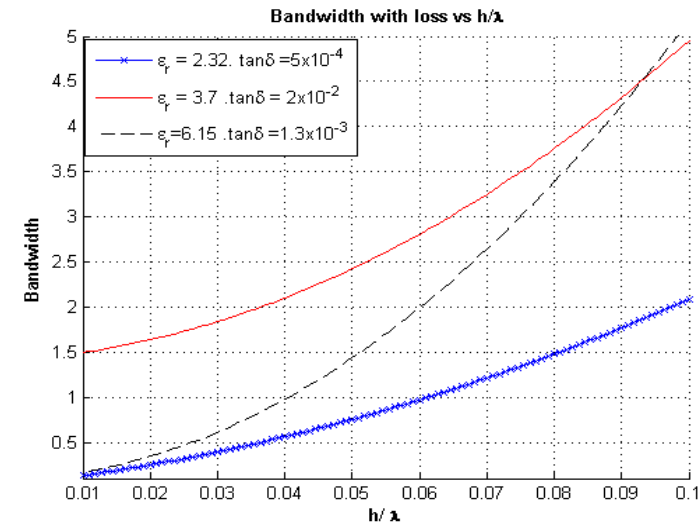

Figure 7 (a). Bandwidth eqn.(12) as a Function of Normalized Substrate Thickness $\left(\left(\frac{h}{a} \geq 0.1\right)\right.$ with Dielectric Losses

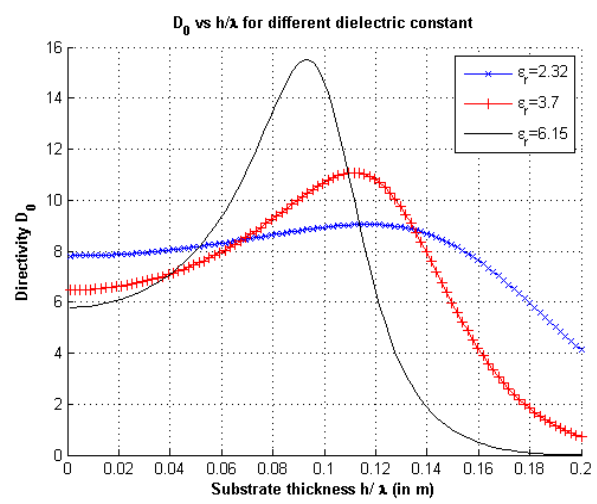

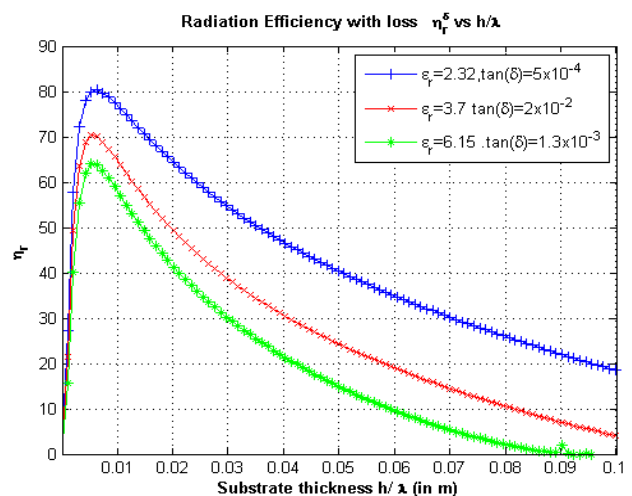

Figure 6(b). Radiation Efficiency eqn.(11) as a Function of Normalized Substrate Thickness (h) Accounting Dielectric Losses

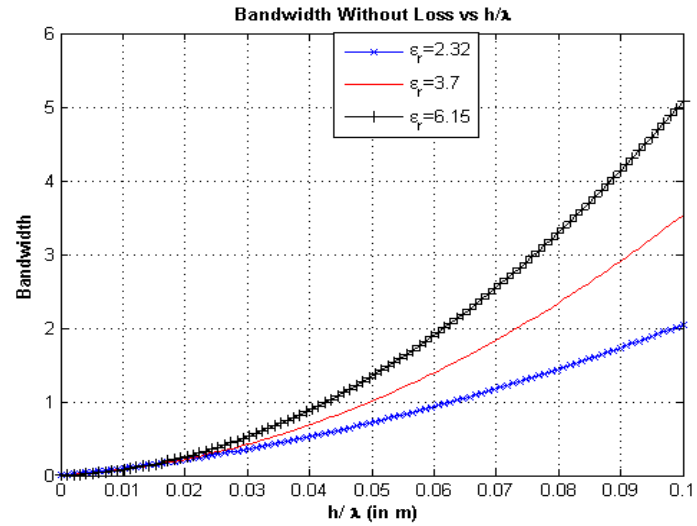

Figure 7 (b). Bandwidth eqn. (12a) as a Function of Normalized Substrate Thickness $\left(\left(\frac{h}{2} \geq 0.1\right)\right.$ without Accounting Dielectric Losses

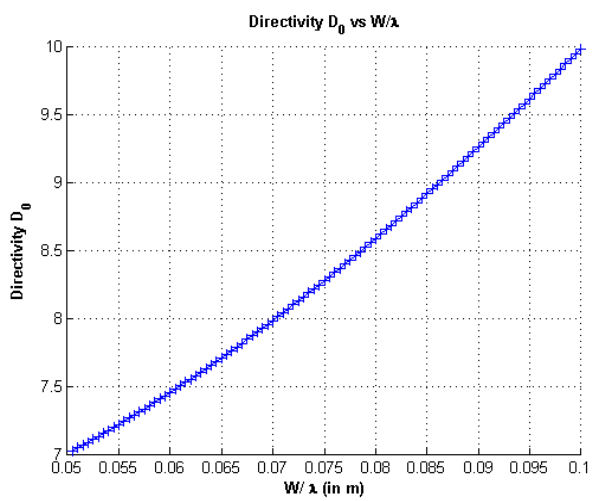


Figure 8 (a). Directivity eqn. (13) as a Function of Normalized Substrate Thickness (h) for Three Different Dielectric Materials
Figure 8 (b). Directivity eqn.(14) as

Function of Normalized Patch Width

$\left(W / \lambda_{0}\right)$

Table 3 gives the comparison of $\mathbf{B W}$ and directivity while Table 4 gives the comparison of directivity for different substrate height.

Table 3. Comparison of Bandwidth and Directivity for Substrate Height $\mathbf{h}$ (1.9 mm -3.1 mm), Effective Width $\left(W_{e}\right)$ and Length $\left(L_{e}\right)$ for Different Dielectric Constant $\left(\epsilon_{r}\right)$ and Loss Tangent $(\tan \delta)$

\begin{tabular}{|l|l|l|l|l|l|l|l|}
\hline $\begin{array}{l}\text { SL. } \\
\text { No. }\end{array}$ & $\in_{\boldsymbol{r}}$ & $\tan \delta$ & $\mathbf{h ~ m m}$ & $W_{e} \mathbf{m m}$ & $L_{e} \mathbf{m m}$ & BW\% eqn.(12) & BW\% eqn.(12a) \\
\hline 1 & 2.32 & $\mathbf{0 . 0 0 0 5}$ & 3.1 & 79.1 & 54.1 & 2.303 & 2.366 \\
\hline 2 & 3.7 & 0.02 & 2.5 & 77.8 & 52.8 & 0.7589 & 2.2078 \\
\hline 3 & 6.15 & 0.0013 & 1.9 & 76.9 & 51.9 & 0.2365 & 0.3731 \\
\hline
\end{tabular}

Table 4. Comparison of Directivity for Substrate Height h $(1.9 \mathrm{~mm}-3.1 \mathrm{~mm})$ Effective Width $\left(W_{e}\right)$ and Length $\left(L_{e}\right)$ for Different Dielectric Constant $\left(\epsilon_{r}\right)$ and Loss Tangent $(\tan \delta)$;

\begin{tabular}{|l|l|l|l|l|l|l|l|}
\hline $\begin{array}{l}\text { SL. } \\
\text { No }\end{array}$ & $\in_{r}$ & $\tan \delta$ & $\mathbf{h ~} \mathrm{mm}$ & $W_{e} \mathrm{~mm}$ & $L_{e} \mathrm{~mm}$ & $\begin{array}{l}D_{0} \\
\text { Eqn.(13) }\end{array}$ & $\begin{array}{l}D_{0} \\
\text { Eqn,(14) }\end{array}$ \\
\hline 1 & 2.32 & $\mathbf{0 . 0 0 0 5}$ & 3.1 & 79.1 & 54.1 & $\mathbf{8 . 0 4}$ & 4.26 \\
\hline 2 & 3.7 & $\mathbf{0 . 0 2}$ & 2.5 & 77.8 & 52.8 & 6.70 & 4.22 \\
\hline 3 & 6.15 & $\mathbf{0 . 0 0 1 3}$ & 1.9 & 76.9 & 51.9 & 5.93 & 4.20 \\
\hline
\end{tabular}

\section{Conclusions}

Based on the theoretical and simulated analysis of the rectangular microstrip antenna with three dielectric substrates, we have calculated the size and design parameters. We have simulated three antennas that can operate at $3 \mathrm{GHz}$ frequency and calculated its radiation efficiency, BW and directivity. Through theoretical and simulated analysis it is observed that the BW increases with increase in substrate thickness and decrease in dielectric constant, while directivity strongly depends on width of the patch. We have simulated fractional change in resonant frequency with the finite size GP and observed the optimal GP dimensions. The printed circuit design presented here is versatility in terms of resonant frequency and polarization purity and low production cost, and is likely to be the most suitable antenna for communication system.

\section{Acknowledgements}

Authors are thankful to the Head, Department of Electronics and Communication Technology, Gauhati University for providing valuable suggestions and necessary computing facilities at the laboratory during the work

\section{References}

[1] G. A. Deschamp, Microstrip microwave antennas, presented at the Third USAF Symposium on Antennas (University of Illinois, USA) (1953).

[2] H. Gutton and G. Baissinot, "Flat aerial for ultra-high frequencies", French Patent No. 703113 (1955). 
[3] R. E. Munson, "Conformal microstrip antennas and microstrip phased arrays", IEEE Trans Antennas Propagation., vol. 22, (1974), pp.74.

[4] J. Q. Howell, "Microstrip antenna", IEEE Trans. Antennas Propag., vol. 23, (1975) pp. 90.

[5] A. G. Derneryd, "A Theoretical Investigation of the Rectangular Microstrip Antenna Element," IEEE Trans. Antennas Propagat., vol. AP-26, no. 4, (1978), pp. 532-535.

[6] K. R. Carver and J. W. Mink, "Microstrip antenna technology", IEEE Trans. Antennas Propag., vol. 29 (1981), pp. 2.

[7] J. L. Kerr, "Microstrip polarization techniques", in Proceedings of the Antenna Applications Symposium (Allerton Park, IL) (1978).

[8] J. Huang, "Microstrip antennas for commercial applications", D. M.Pozar and D. H. Schaubert, (Eds.), IEEE (Piscataway, NJ), pp. 371 (1995).

[9] R. K. Vishwakarma and S. Tiwari, "Dual-band stacked rectangular microstrip antenna for mobile applications”, School of Studies in Electronics Pt. Ravishankar Shukla University, (2010), pp. 1.

[10] T. Fukusako and H. Maema, "Radiation Efficiency of Low-profile and Electrically Small Printed Antennas with Capacitive Feeding Structure", Progress in Electromagnetic Research Symposium (Sweden), (2013), pp. 515.

[11] C. A. Balanis, “Antenna Theory: Analysis and Design (Wiley-Interscience)”, (2005), pp. 810-817.

[12] R. Garg, P. Bhartia, I. Bahl and A. Ittipiboon, "Microstrip Antenna Design Handbook (Artech House)", (2001), pp. 285.

[13] G. Kumar and K. P. Ray, "Broadband Microstrip Antenna (Artech House)”, (2003), pp. 383.

[14] K. F. Lee and W. Chen, "Advances in Microstrip and Printed Antennas (Wiley)”, (1997), pp. 223-264.

[15] D. R. Jackson and N. G. Alexopoulos, "Simple Approximate Formulas for Input Resistance, Bandwidth, and Efficiency of a Resonant Rectangular Patch", IEEE Trans. Antennas Propagat., vol. 39, no. 3, (1991), pp. 407-410.

[16] K. J. Vinoy, "Multifunctional Microstrip Antennas for Wireless Applications", IEEE Applied Electromagnetics Conference, (2011) December.

[17] R. D. Kanphade, D. G. Wakade and N. T. Markad, "Microstrip Rectangular Patch Antenna : Computer Aided Design Methodology" International Journal of Computer Science and Information Technologies, vol. 3, no. 1, (2012), pp. $3144-3146$.

\section{Authors}

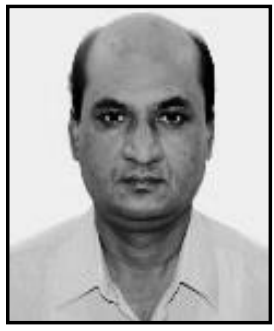

Tulshi Bezboruah received the B.Sc. degree in physics with electronics under the University of Dibrugarh, Dibrugarh, India, in 1990, and the M.Sc. and Ph.D. degrees in electronics and radio physics from Gauhati University, Guwahati, India, in 1993 and 1999, respectively. In February 2000, he joined in the Department of Electronics Science, Gauhati University, as a Lecturer. He is currently the Professor \& Head, Department of Electronics and Communication Technology, Gauhati University. His current research interest include web based instrumentation and control, web service, and communication systems. Professor Bezboruah is member of the IEEE, IEEE Geoscience and Remote Sensing Society as well as an Associate Member of the International Center for Theoretical Physics, Trieste, Italy.

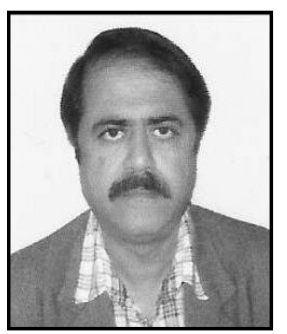

Prabir Banerjee received the BSc degree with honors in Physics under Gauhati University, Guwahati, India in 1984, and M. Sc (Electronics) degree from Delhi University in 1988. In November 1989 he joined Department of Physics, Jagiroad College, Jagiroad, Morigaon. Assam, India as a Lecturer, he is currently holding the post of Associate Professor in Physics in the same college. His research interest includes Microstrip Antennas and communication technology. 